\title{
A Pure NanolCG-Based Homogeneous Lipiodol Formulation: Toward Precise Surgical Navigation of Hepatocellular Carcinoma After Long-Term Transcatheter Arterial Embolization
}

\author{
Yang Zhang \\ Xiamen University \\ Hongwei Cheng \\ Xiamen University \\ Hu Chen \\ Xiamen University \\ Peiyao Xu \\ Huaqiao University
}

En Ren

Xiamen University

Yonghe Jiang

Xiamen University

Dengfeng Li

Xiamen University

Xing Gao

Xiamen University

Yating Zheng

Xiamen University

Pan He

Xiamen University

Huirong Lin

Xiamen University

Biaoqi Chen

Huaqiao University

Gan Lin

Xiamen University

Aizheng Chen

Huaqiao University

Chengchao Chu

Xiamen University 


\section{Jingsong Mao}

Xiamen University

Gang Liu ( $\sim$ gangliu.cmitm@xmu.edu.cn )

Xiamen University https://orcid.org/0000-0003-2613-7286

\section{Research Article}

Keywords: hepatectomy, carcinoma, nanoparticles, homogeneous

Posted Date: September 8th, 2021

DOl: https://doi.org/10.21203/rs.3.rs-860171/v1

License: (c) (1) This work is licensed under a Creative Commons Attribution 4.0 International License. Read Full License 


\section{Abstract}

Purpose To surmount the critical issues of clinical hepatectomy and achieve a precise surgical navigation of hepatocellular carcinoma after long-term transcatheter arterial embolization (TAE).

Methods A facile and green pure-nanomedicine formulation technology was developed to construct carrier-free indocyanine green nanoparticles (nanoICG). The nanoICG was dispersed into lipiodol via a super-stable homogeneous lipiodol formulation technology (SHIFT nanoICG) for TAE combined with near-infrared fluorescence-guided precise hepatectomy.

Results We demonstrate that SHIFT nanoICG integrates excellent anti-photobleaching capacity, great optical imaging properties, and specific tumoral deposition to recognize tumor regions, featuring sufficient physical stability for fluorescence-guided precise hepatectomy. Importantly, SHIFT nanoICG is capable of visualizing all lesions in rabbit-bearing VX2 orthotopic hepatocellular carcinoma models, even the tiny focus to $0.6 \times 0.4 \mathrm{~mm}$.

Conclusion Our findings indicate that SHIFT nanoICG provides a promising avenue to address the clinical issue of hepatectomy and has excellent potential for a translational pipeline.

\section{Introduction}

Hepatocellular carcinoma (HCC) is the fifth most prevalent malignancy worldwide and is characterized by poor prognosis and high mortality [1]. Despite the slew of emerging strategies [2-5], surgical resection remains the mainstream treatment for HCC [6, 7]. However, the efficiency of surgical resection has been compromised by ill-defined tumor margins and occult lesions of HCC [8]. Consequently, aggressive hepatectomy tends to lower prognosis, while conservative resection can easily lead to relapse. In clinical settings, fluorescence-guided surgical navigation is promising to achieve specific operations, realizing an excellent prognosis. Indocyanine green (ICG), the only Food and Drug Administration (FDA) approved near-infrared fluorescent agent, has been widely applied in the surgical navigation of solid tumors and metastases, showing low toxicity and prominent optical visualization $[9,10]$. Despite its advantages, ICG suffers from three critical issues: i) rapid clearance in blood circulation; ii) instability and poor light bleaching resistance; and $\rrbracket$ ) lack of tumor specificity. Therefore, it is imperative to overcome the issues of ICG and achieve a stably entire surgical-span procedure imaging-guided specific hepatectomy.

Advances in nanotechnologies and biomaterial sciences have successfully promoted the fluorescent properties and in vivo metabolism of ICG, thereby extending its biomedical applications. For example, both nanovesicle-loaded and chemically synthesized ICG-based nanoparticles demonstrated a long blood circulation and stability for tumor-specific imaging [11-14]. However, additional ingredients were inevitably required for the preparation of ICG nanoparticles, which may introduce toxicity and increase the complexity of clinical translation. Recently, many pure nanomedicines have been fabricated using the supercritical fluids antisolvent (SAS) strategy with the help of supercritical carbon dioxide $\left(\mathrm{SC}^{-} \mathrm{CO}_{2}\right)[15$, 16]. SAS enables the self-assembly of clinical medicines without any additive ingredients or solvents; it 
may, thereby, be useful in further studies of pure nanomedicines. This environmentally-friendly strategy is regarded as a super-stable pure-nanomedicine formulation technology (SPFT) and broadens the potential clinical applications for nanomedicines.

In clinical treatment, some HCC patients are suitable for direct surgical treatment [17]; performing the surgery following transcatheter arterial embolization (TAE) was a critical combined strategy for patients with advanced HCC [18-20]. Lipiodol is the most common embolic agent and exerts an excellent curative effect of tumor-feeding artery embolization and better biological safety, which benefited from the specific uptake of lipiodol by tumor cells and in situ drug administration [21, 22]. In addition, fluorescent iodized emulsions could accomplish imaging-guided surgical navigation after TAE [23, 24]. However, two issues should be resolved before identifying an ideal fluorescence-labeled embolic candidate: i) homogeneous dispersion and long-time stability; and ii) high fluorescence intensity and accurate identification of tumor boundaries. In our previous study, we constructed a super-stable homogeneous intermixed formulation technology (SHIFT), in which the drug molecule could disperse homogeneously in lipiodol $[25,26]$.

Hereby, we develop a carrier-free ICG nanoparticle based homogeneous lipiodol formulation (SHIFT nanoICG) for precise surgical treatment after TAE. We found that SHIFT nanoICG performs an excellent tumor-specific deposition after TAE, and thereby enables nanoICG to visualize the tumor regions for precise resection (Fig. 1). The prepared SHIFT nanoICG exhibited superior retention in lesions and stable fluorescence imaging capability. In particular, SHIFT nanoICG shows great anti-photobleaching ability under $6 \mathrm{~h}$ of continuous irradiation from a fluorescent navigation system, allowing fluorescence imaging that endures throughout the entire surgically guided resection. As a result, SHIFT nanoICG exhibits remarkable retention in the rabbit ear-bearing VX2 tumor model and is capable of visualizing all lesions in the VX2 orthotopic hepatocarcinoma model, even the tiny focus to $0.6 \times 0.4 \mathrm{~mm}$. Therefore, SHIFT nanoICG is a promising multifunctional formulation for HCC resection and may prove valuable for clinical translation efforts in cancer therapy.

\section{Materials And Methods}

\section{Materials}

Indocyanine green was purchased from Aladdin Biochemical Technology Co., Ltd. Lipiodol was purchased from Jiangsu Hengrui Pharmaceutical Company. Pentobarbital sodium was purchased from Lulong biotechnology Co., Ltd. Dichloromethane and ethyl alcohol were purchased from Sigma-Aldrich Biochemical Technology Co., Ltd. Our lab developed the SHIFT and SPFT equipment (Patent no. 2019107349374.4; 201910105683X and 16581600), Xiamen University. Interventional instruments were available from the radiology department of Xiang'an Hospital of Xiamen University.

\section{Synthesis and characterization of nanoICG}

A total of $20 \mathrm{mg}$ ICG molecule was dissolved into $10 \mathrm{~mL}$ of ethyl alcohol and dichloromethane mixed solution, and then the mixed solution was dropped into a supercritical $\mathrm{CO}_{2}$ fluid reactor along with a 
speed of $1 \mathrm{~mL} / \mathrm{min}$. After 30 min reaction, the solvent was removed by supercritical $\mathrm{CO}_{2}$, resulting in the precipitation of nanoICG.

Then, the collected nanoICG was measured by SEM (EM CPD300, Leica, German), and DLS (Malvern Instruments, Malvern, UK). The fluorescent and photoacoustic signal intensity were detected through IVIS

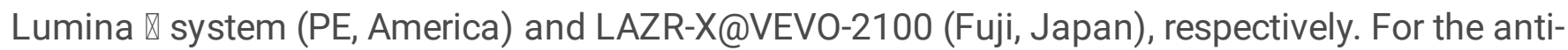
photobleaching capacity detection of nanoICG, free ICG and nanoICG were irradiated by $808 \mathrm{~nm}$ laser (1 $\left.\mathrm{W} / \mathrm{cm}^{2}\right)$ for different time points $(5,10 \mathrm{~min})$, and then the fluorescent signal was following measured by IVIS Lumina $₫$ system.

\section{Synthesis and characterization of SHIFT nanoICG}

In the homogeneous dispersion process, $2 \mathrm{mg}$ nanoICG was comingled with $10 \mathrm{~mL}$ lipiodol in the reaction device. The $\mathrm{CO}_{2}$ gas was developed to supercritical fluid at the condition of $55^{\circ} \mathrm{C}$ with $1500 \mathrm{rpm}$ stirring speed and $20 \mathrm{MPa}$ via a cyclic pressure pump. After 90 min reaction, the sample was collected along with pressure relief, and the sample was named SHIFT nanoICG.

For the characterization, nanoICG was re-extracted from SHIFT nanoICG $(300 \mu \mathrm{L})$ via saline $(600 \mu \mathrm{L})$ at $37^{\circ} \mathrm{C}$ for different time points $(1,2,3,5$, and 7 days). UV-2100 spectrophotometer (Shimadzu, Japan), TEM (TecnaiG2 Spirit, FEI), and LC-MS (SPD-20A, Japan) were used to verify the molecular component of nanoICG. The viscosities of lipiodol, the mixture of nanoICG and lipiodol, and SHIFT nanoICG were detected with viscosimeter at $37^{\circ} \mathrm{C}$. Radio-imaging properties of lipiodol and SHIFT nanoICG were investigated on the CT system (Siemens Inveon micro-CT instrument).

\section{Preparation of decellularized liver and determination of drugs release}

The decellularized liver model was constructed by a previously reported method [27]. Briefly, the rat liver was intactly removed and frozen at $-80^{\circ} \mathrm{C}$ overnight. Then, the portal vein and inferior vena cava of liver were irrigated via $5 \%$ sodium dodecyl sulfate (SDS) solution with a flow velocity of $4 \mathrm{~mL} / \mathrm{min}$ for 8 to 10 hours after organ thawing, and then rinsed with $0.9 \%$ saline to remove SDS.

For agent evaluation, nanolCG solution was prepared with a concentration of $2 \mathrm{mg} / \mathrm{mL}$. Then, the prepared decellularized liver models were rinsed with $0.9 \%$ saline to full vessels, and the formulation was slowly injected into the hepatic inferior vena cava with a $1 \mathrm{~mL}$ syringe. After that, fluorescence microscope, stereoscope, PAI, DSA and CT were used to capture images at different time points to investigate the behaviors. The captured images were analyzed and semi-quantified by Image $\mathrm{J}$ software (National Institute of Health, Bethesda, MD, USA).

\section{VX2 ear-bearing subcutaneous and orthotopic liver cancer model of rabbit}

Animal experiments were approved by Animal Care and Use Committee (CC/ACUCC) of Xiamen University. New Zealand white rabbits $(2.5-3.0 \mathrm{~kg}$ ) were obtained from Shanghai SLAC Laboratory Animal. VX2 tumor mass (Shanghai Lalan Biotechnology) was planted in the hind leg of rabbit. After 15 
days, the active VX2 tumor was obtained and cut into $1 \mathrm{~mm}^{3}$ piece to implant into rabbit ear for subcutaneous models and left liver for orthotopic models, respectively. For the subcutaneous models, the tumor could reach 100-150 $\mathrm{mm}^{3}$ after 7 days, and for the orthotopic models, the tumors were verified via 3.0 T MRI (Magnetom Skyra) after 15 days.

\section{Fluorescence-guided orthotopic surgical hepatectomy}

When the orthotopic VX2 tumor size reaches about $10 \mathrm{~mm} \times 10 \mathrm{~mm}$, the rabbits were subjected to take intervention through a percutaneous intra-arterial femoral port-catheter system by the guidance of DSA, and then SHIFT nanoICG ( $200 \mu \mathrm{L}, 2 \mathrm{mg} / \mathrm{mL}$ ) was injected into lesions slowly. After that, the evaluation of intervention was investigated via CT plain scan. After 7 days, the anesthetic rabbits were submitted to implement fluorescence-guided navigation surgical resection. After disinfected by iodophor, the liver was exposed. The fluorescent surgical navigation system (Wuxi Apptec, China) was applied to lighten the liver under dark and sterile condition and observe lesions, a high-signal region, through the display screen of the system. And then the contrast mode of the equipment could use to ensure the boundary between tumor and normal liver tissue. Afterward, the tumor lesions could be resected under the guidance of the lighted area on the screen. Subsequently, the resected tissues were investigated by PAI, and DR to verify the signal of nanoICG and lipiodol. Finally, H\&E staining was utilized to evaluate the resection.

\section{Biological safety test}

On 7th day, we collected about $200 \mu \mathrm{L}$ blood of the embolic rabbits from the ear vein, and alanine transaminase (ALT), alkaline phosphatase (ALP), aspartate aminotransferase (AST), albumin (ALB), creatine kinase (CK), and urease (UREA), the indicators of renal, cardiac, and liver function were tested by the corresponding kit.

\section{Results}

\section{Synthesis and characteristics of nanoICG via SPFT}

To synthesize nanoICG, we designed a pure physical nanomedicine technology. In brief, ICG molecules were dissolved into a low boiling point solvent of ethyl alcohol and dichloromethane solution, and the mixed solution was then dropped into $\mathrm{SC}-\mathrm{CO}_{2}$ fluid reaction slowly and equably. After pressure relief, the solvent was removed via pneumatolysis of $\mathrm{CO}_{2}$, and the uniform pure ICG nanoparticle was obtained. Scanning electron microscopy (SEM) revealed that nanoICG particles were homogeneous spherical particles (Fig. 2a), and dynamic light-scattering (DLS) analyses confirmed the hydrate particle size of nanolCG to be $40.7 \pm 4.5 \mathrm{~nm}$ (Fig. 2b) with a low particle dispersion index (PDI) of 0.163 , which testifies to the excellent biomedical application potential of nanoICG.

\section{Optical imaging performance of nanolCG}


The ultra-violet (UV) absorption of nanoICG was then investigated. The UV absorption curves implied that the absorption of nanolCG did not differ significantly from that of free ICG (Fig. 2c). Owing to the photoacoustic (PA) imaging capability of ICG $[28,29]$, we tested the PA imaging ability of nanoICG, revealing that nanoICG exhibited higher PA intensity up to three times higher than free ICG (Fig. 2d, e).

Subsequently, the photothermal (PT) conversion capacity of nanolCG was investigated (Fig. S1). The result indicated that PT conversion of nanoICG performed a faster heating speed than free ICG under 808nm-wavelength irradiation. The PA and PT properties of nanoICG suggested that the thermal expansion coefficient of the ICG molecule has been improved due to the nano-crystallization via SPFT. Additionally, nanoICG performed outstanding anti-photobleaching capability, a critical property for surgical navigation [30], which enables much higher photostability than that of free ICG (Fig. 2f, g). It is also notable that the fluorescence of nanoICG recovered along with irradiation at a wavelength of $808 \mathrm{~nm}\left(1 \mathrm{~W} / \mathrm{cm}^{2}\right)$, and even exhibited a pH dependence in that the fluorescence intensity was enhanced with a mildly acid solution (pH 6.5) than with a neutral solution (Fig. S2). Therefore, we inferred that nanoICG has higher optical imaging capacity and anti-photobleaching capability than free ICG molecules and may help achieve enhanced surgical navigation.

\section{Synthesis and characteristics of lipiodol-nanoICG formulation via SHIFT}

For the further application of nanoICG in TAE and fluorescence-guided surgical navigation combined strategy, we commingled nanoICG into lipiodol homogeneously utilizing our previously reported SHIFT strategy [25] (Fig. 3a). Firstly, nanolCG was released into saline to verify whether the morphology and property were affected via SHIFT. The released experiment of SHIFT nanoICG was tested at pH 6.5 and $\mathrm{pH} 7.4$ at $37{ }^{\circ} \mathrm{C}$, respectively. The result exhibits that the release rate of SHIFT nanoICG in saline ( $\mathrm{pH} \mathrm{6.5)}$

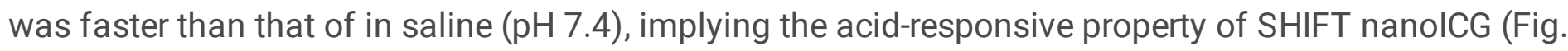
S3). Transmission electron microscopy (TEM) revealed that nanoICG remained nano-sized (diameter: $30 \mathrm{~nm}$, Fig. 3b), and the UV absorption curve showed that the absorption peak of SHIFT nanoICG was broader than the absorption peak of released nanoICG, which showed the same absorption curve as free ICG (Fig. 3c). Furthermore, the molecular structure of nanoICG in lipiodol was determined by liquid chromatography-mass spectrometry (LC-MS), which indicated the molecular component consistency of nanoICG in lipiodol relative to the ICG standard samples (Fig. 3d). Interestingly, the retention time (RT) of nanoICG was shorter than that of the ICG standard, suggesting that nanoICG may have a different physical polarity state than free ICG.

Maintaining viscosity is crucial for lipiodol to realize effective embolization. Thus, the viscosity of SHIFT nanoICG was compared with that of lipiodol [31], revealing no obvious changes in viscosity following the SHIFT procedure (Fig. 3e). SHIFT nanoICG also exhibited admirable stability at room temperature even up to 60 days (Fig. 3f). These results demonstrated that SHIFT nanoICG kept the properties of nanoICG and lipiodol compatibility, and this green technology, which does not require additional chemicals, is better for clinical translation. 
To further evaluate the imaging performance of SHIFT nanoICG, we first investigated its fluorescent imaging capability. As expected, SHIFT nanoICG exhibited a higher fluorescence intensity at pH 6.5 than at pH 7.4, even at the 7-day timepoint (Fig. 3g), indicating that SHIFT nanolCG may exert long-term and specific fluorescence at the tumor microenvironment (TME) [32]. In addition, PA imaging showed that SHIFT nanoICG not only had an excellent signal which was approximately 20 times and 3 times higher than that of free ICG and nanoICG, respectively, but also had most homogenous signal than the other groups, which indicates its outstanding imaging ability (Fig. 3h and Fig. S4). Because, lipiodol could act as contrast agent in computerized tomography (CT) imaging, we measured the CT imaging performance of SHIFT nanoICG. The results showed that the CT imaging performance of SHIFT nanoICG was consistent with that of lipiodol and lasted for 60 days with no obvious changes (Fig. 3i). All of this evidence demonstrated the profound value of SHIFT nanoICG in promoting ICG performance, especially for precise tumor imaging. SHIFT nanoICG has been shown to effectively overcome the weak antiphotobleaching ability of ICG and to have a superior imaging capacity for surgical navigation.

\section{Evaluation of SHIFT nanoICG formulation in a decellularized liver model}

Decellularized liver is a meaningful model to investigate the stability, homogeneity, drug release properties, and embolic effects of SHIFT nanoICG. Firstly, as shown in Fig. 4a and Fig. S5, the decellularized liver model was successfully established. We demonstrate that the pure water-soluble nanolCG solution injected into the decellularized liver venous was diffused out of the blood vessels in only $30 \mathrm{~min}$ (Fig. S6), while the lipiodol-based formulation did remain relatively stable. Twenty-four hours

after the injection, SHIFT nanoICG group showed an excellent homogenous distribution and imperceptible drug release, while the Mix nanoICG group exhibited obvious aggregation and nanolCG release from vessels (Fig. 4b). PA imaging was also used to investigate the behavior of nanolCG, revealing that the signal of SHIFT nanoICG remained nearly unchanged, while the signal of Mix nanolCG was heterogeneous and concentrated in the vessel wall (Fig. 4c and S7), indicating SHIFT nanolCG has a stable and homogeneous distribution in blood vessels. Furthermore, three-dimensional (3D) confocal microscopy evaluated the distribution and drug release behavior of Mix nanolCG and SHIFT nanoICG after injection into the venous system of the decellularized liver model. The results showed that SHIFT nanoICG can disperse into tiny blood vessels and displays a stable and uniform morphology, while Mix nanoICG was nearly diffused out of the blood vessels within $72 \mathrm{~h}$ (Fig. 4d, e). Moreover, digital subtraction angiography (DSA) and CT-3D imaging showed that lipiodol could embolize the blood vessels perfectly, even within $72 \mathrm{~h}$ (Fig. 4f). These results suggest that SHIFT nanolCG has excellent stability, dispersibility, and slow release in the liver, which testifies to its promising applications in vivo.

\section{Evaluation of the navigation performance of SHIFT nanoICG in rabbit ear-bearing subcutaneous VX2 models}

On account of the favorable behavior of SHIFT nanoICG in optical imaging, anti-photobleaching, TMEresponsive release, and the preservation of lipiodol attributes, the evaluation of SHIFT nanoICG was further investigated in rabbit ear-bearing subcutaneous VX2 models. As illustrated in Fig. 5a, the primary 
VX2 subcutaneous models were constructed on rabbit ears, and after approximately 7 days, the successfully constructed models were randomly divided into one of three groups: mixed nanoICG, SHIFT ICG, and SHIFT nanoICG ( $n=3$, respectively). Under the guidance of DSA, the drugs $\left(C_{I C G}=0.2 \mathrm{mg} / \mathrm{mL}\right.$, $200 \mu \mathrm{L}$ ) were accurately embolized into lesions and subsequently visualized by a fluorescence-guided surgical navigation system for point-in-time monitoring ( $3 \mathrm{~h}, 1,3,7,14$, and 21 days) to assess the performance of navigation agents. The fluorescence results showed that SHIFT nanoICG not only fluorescently marked the tumor lesion precisely, but the fluorescence also lasted over the long-term (up to 21 days) with high intensity (Fig. 5b). Conversely, despite the fact that the SHIFT ICG group also illustrated good fluorescent navigated attribution, the fluorescence signal declined continuously after post-surgical day 1 . Furthermore, the semiquantitative fluorescent analysis demonstrated that the strongest signal of SHIFT nanolCG was almost twice as high as that of SHIFT ICG (Fig. 5c), suggesting that SHIFT nanoICG acted as a better navigation agent for embolization.

Subsequently, digital radiography (DR) imaging was used to verify whether the lipiodol deposited into tumor lesions after 21 days, revealing that lipiodol was still retained after 21 days in all groups (Fig. 5d). The PA imaging of the resected tumor lesions illustrated that the SHIFT nanoICG and SHIFT ICG groups had a higher PA intensity than the Mix nanoICG group (Fig. 5e, f), suggesting that SHIFT is a superior technology for controlled drug release. The biochemical indexes of the liver, kidney, and heart function were further estimated, including alanine transaminase (ALT), alkaline phosphatase (ALP), aspartate aminotransferase (AST), albumin (ALB), creatine kinase (CK), and urease (UREA). The results showed that the indexes had nearly no obvious change compared to the control group (Fig. S8). As such, SHIFT nanoICG is a remarkable candidate for precise fluorescence-guided surgical navigation via TAE.

\section{SHIFT nanoICG for precise surgical navigation of orthotopic HCC}

Based on the excellent fluorescence performance and super-stable deposition of SHIFT nanoICG, the fluorescence-guided surgical navigation capacity of SHIFT nanoICG in vivo was proposed and validated (Fig. 6a). The primary VX2 orthotopic HCC models were established, and the successful models were confirmed by MRI (Fig 6b). Owing to the side-effects of high ICG concentration (up to $0.5 \mathrm{mg} / \mathrm{kg}$ ), including fever, shock, and allergy [33-35], the working concentration of nanolCG was investigated via intravenous injection. Delightfully, nanoICG exhibited a superior fluorescent ability relative to free ICG at a low concentration of $0.08 \mathrm{mg} / \mathrm{kg}$ (Fig. S9). Consequently, under the guidance of DSA, the SHIFT nanoICG was taken to achieve tumor embolization, as shown in Fig. 6c. Afterward, CT imaging confirmed that SHIFT nanoICG was fully deposited in the tumor area (Fig. 6d), and the CT value of the lipiodol deposition area (LDA) and the liver parenchymal area (LPA) were also evaluated (Fig. S10). The results indicated that SHIFT nanoICG was homogeneously dispersed into the tumor area, with very little deposited in healthy liver tissue, suggesting that SHIFT nanoICG is an excellent agent for TAE.

Due to the complexity and difficulty of performing an effective HCC surgery, this surgery invariably requires a long operating time [36]; thus, the fluorescence-guided hepatectomy agent needs excellent fluorescent stability to persist throughout the entire duration of the operation. Therefore, after 7 days of 
TAE, the embolic lesion was exposed under the irradiation of a fluorescent emitter for 6 continuous hours. As expected, the fluorescence of SHIFT nanoICG not only clearly exhibited the tumor region and margin, but also remained at over $80 \%$ of the initial intensity after $6 \mathrm{~h}$ of irradiation (Fig. 6e), indicating that SHIFT nanoICG has great potential to guide an entire complex hepatectomy with high precision in vivo. As expected, the tumors with SHIFT nanoICG were visualized with clear tumor delineation, establishing an excellent condition for guiding hepatectomy (Fig. 6f). In particular, after resecting the obvious lighting tumors, we re-evaluated the liver tissue and observed an additional two tiny light spots, which indicated the presence of residual lesions. Subsequently, hematoxylin and eosin (HE) staining of resected tissues demonstrated that SHIFT nanoICG-lightened tissues were tumor lesions, and the smallest re-assessed tumor was as little as $0.6 \mathrm{~mm} \times 0.4 \mathrm{~mm}$ (Fig. $6 \mathrm{~g}$ ). The results suggested that SHIFT nanolCG possesses ultra-high sensitivity for tumor recognition to guide a precise hepatectomy and to preserve as much normal liver tissue as possible. Additionally, HE staining delineated the margin between tumor and liver tissues clearly, and the normal tissues showed no obvious damage caused by SHIFT nanoICG. The liver, kidney, and heart function were further assessed through the bio-indicators of ALT, ALP, AST, ALB, CK, and UREA. The results illustrated that the liver, kidney, and heart functions were only slightly affected relative to the healthy control group (Fig. 6h), demonstrating that SHIFT nanoICG has excellent biocompatibility, safety, and potential for clinical translation.

\section{Discussion}

Despite the fact that fluorescence-guided surgical navigation of $\mathrm{HCC}$ is a promising method to realize high-precision resection for a good prognosis, ICG suffers from several critical issues, including rapid metabolism, weak anti-photobleaching ability, and no targeting capacity. Therefore, developing a highperformance agent for high-precision hepatectomy is strongly desired. In this study, we provide a facile, green, and economical strategy of super-stable pure-nanomedicine formulation technology (SPFT) to produce nanoICG, and which dispersed in lipiodol through our constructed super-stable homogenous lipiodol formulation technology (SHIFT), with an ultra-high stability for a period exceeding 60 days. We demonstrate that nanoICG has better optical properties than free ICG, and that nanolCG combines with the excellent specific tumoral deposition of lipiodol to achieve precise and enduring guided hepatectomy, thereby significantly improving the visualization of otherwise indiscoverable satellite lesions. In particular, based on the favorable optical capacity of nanoICG and suitable technology, the working concentration of nanolCG is as low as $0.08 \mathrm{mg} / \mathrm{kg}$, which is approximately one-seventh of the potential toxic concentration of ICG. Therefore, SHIFT nanoICG could overcome the current clinical challenges to achieving precise $\mathrm{HCC}$ identification and resection.

Furthermore, SPFT is a green physical process and the characteristics of uniform size, clear components, and easily scalable production endows nanoICG with great clinical translational potential. Importantly, SHIFT is also a physical dispersion method that has excellent performance, safety, and effectiveness [25], and that has been used in clinical multicenter clinical trials (Register No.ChiCTR2000035055, data not shown). Taken together, SHIFT nanoICG is an effective, green, and safe fluorescence-guided embolic agent for precise surgical navigation and is suitable for the translational pipeline. Moreover, the SPFT 
could be extended to chemotherapeutics, such as paclitaxel, and oxaliplatin, among others, for enhanced chemotherapy. Additionally, chemotherapeutic agents and the ICG molecule could also be developed as a kind of homogeneously mixed nanoparticles via SPFT, and could both be combined with TAE to achieve more effective therapy, especially for that of advanced HCC. These chemotherapeutic agents and embolization cooperated to reduce tumor volume, after which ICG can guild a precise resection to improve prognosis. Such efforts are now underway in our laboratory.

\section{Conclusion}

In summary, the facile and green SHIFT nanoICG technology integrates the excellent anti-photobleaching ability and imaging sensitivity of pure nanoICG, and the specific tumoral deposition of lipiodol, to address the clinical issues of fluorescence-guided surgical navigation to realize a precise fluorescence-guided hepatectomy, even for satellite lesions. Given the remarkable biocompatibility and biosafety of ICG and lipiodol, SHIFT nanoICG has great potential for clinical hepatectomy and other cancer therapy.

\section{Declarations}

\section{Acknowledgements}

We thank Xiaoyong Wang, Zicheng Huang, Shanshan Meng, Dongbei Guo, and Youliang Yao for technical assistance.

\section{Funding}

This work was supported by the Major State Basic Research Development Program of China (2017YFA0205201), the National Natural Science Foundation of China $(81925019,81422023,81603015$, 81871404, and U1705281), the Fundamental Research Funds for the Central Universities (20720190088 and 20720200019), the Program for New Century Excellent Talents in University, China (NCET-13-0502).

\section{Author contributions}

Y. Z. and G. Liu conceived and designed the experiments. Y. Z., H. Cheng, H. Chen, P. X., E. R., Y. J., X.G., D.L., J. M., and Y. Z. performed the experiments. Y. Z., B.-Q. C., P. H., H. L. and A. C., analyzed the results. Y. Z., H. Cheng, G. Lin, C. C., J. M., and G. Liu wrote the manuscript. G. Liu supervised the entire project.

\section{Conflict of interest}

The authors declare no competing interests.

\section{Ethical approval}

All animal studies followed the international guidelines of animal care and were approved by Animal Care and Use Committee (CC/ACUCC) of Xiamen University. 


\section{References}

1. Siegel R, Miller K, Jemal A. Cancer statistics. 2020. CA Cancer J Clin 2020; 70: 7-30. https://doi.org/10.3322/caac.21590.

2. Shi X, Zhang Y, Tian Y, Xu S, Ren E, Bai S, et al. Multi-responsive bottlebrush-like unimolecules selfassembled nano-riceball for synergistic sono-chemotherapy. Small Methods 2020: 2000416. https://doi.org/10.1002/smtd.202000416.

3. Zhang Y, Wang X, Chu C, Zou Z, Chen B, Pang X, et al. Genetically engineered magnetic nanocages for cancer magneto-catalytic theranostics. Nat Commun. 2020;11:5421. https://doi.org/10.1038/s41467-020-19061-9.

4. Sun T, Han J, Liu S, Wang X, Wang Z, Xie Z. Tailor-made semiconducting polymers for second nearinfrared photothermal therapy of orthotopic liver cancer. ACS Nano. 2019;13:7345-54. https://doi.org/10.1021/acsnano.9b03910.

5. Zhu J, Chu C, Li D, Pang X, Zheng H, Wang J, et al. Fe(III)-porphyrin sonotheranostics: A green tripleregulated ROS generation nanoplatform for enhanced cancer imaging and therapy. Adv Funct Mater. 2019;29:1904056. https://doi.org/10.1002/adfm.201904056.

6. Vibert E, Schwartz M, Olthoff K. Advances in resection and transplantation for hepatocellular carcinoma. J Hepatol. 2020;72:262-76. https://doi.org/10.1016/j.jhep.2019.11.017.

7. Savic L, Schobert I, Peters D, Walsh J, Laage-Gaupp F, Hamm C, et al. Molecular imaging of extracellular tumor $\mathrm{pH}$ to reveal effects of locoregional therapy on liver cancer microenvironment. Clin Cancer Res. 2020;26:428-38. https://doi.org/10.1158/1078-0432.CCR-19-1702.

8. Aufhauser D, Sadot E, Murken D, Eddinger K, Hoteit M, Abt P, et al. Incidence of occult intrahepatic metastasis in hepatocellular carcinoma treated with transplantation corresponds to early recurrence rates after partial hepatectomy. Ann Surg. 2018;267:922-8.

https://doi.org/10.1097/SLA.0000000000002135.

9. Dai Q, Ren E, Xu D, Zeng Y, Liu G. Indocyanine green-based nanodrugs: A portfolio strategy for precision medicine. Prog Nat Sci. 2020;5:577-88. https://doi.org/10.1016/j.pnsc.2020.08.002.

10. Lu H, Gu J, Qian X, Dai X. Indocyanine green fluorescence navigation in laparoscopic hepatectomy: a retrospective single-center study of 120 cases. Surg Today. 2021;51:695-702.

https://doi.org/10.1007/s00595-020-02163-8.

11. Lin H, Li S, Wang J, Chu C, Zhang Y, Pang X, et al. A single-step multi-level supramolecular system for cancer sonotheranostics. Nanoscale Horiz. 2019;4:190-5. https://doi.org/10.1039/c8nh00276b.

12. Lin G, Zhang Y, Zhang L, Wang J, Tian Y, Cai W, et al. Metal-organic frameworks nanoswitch: Toward photo-controllable endo/lysosomal rupture and release for enhanced cancer RNA interference. Nano Research. 2020;13:238-45. https://doi.org/10.1007/s12274-019-2606-2.

13. Zhang P, Zhang L, Qin Z, Hua S, Guo Z, Chu C, et al. Genetically engineered liposome-like nanovesicles as active targeted transport platform. Adv Mater. 2018;30:1705350. https://doi.org/10.1002/adma.201705350. 
14. Chu C, Ren E, Zhang Y, Yu J, Lin H, Pang X, et al. Zinc(II)-dipicolylamine coordination nanotheranostics: Toward synergistic nanomedicine by combined photo/gene therapy. Angew Chem Int Ed Engl. 2019;58:269-72. https://doi.org/10.1002/anie.201812482.

15. Chen B, Kankala R, Zhang Y, Xiang S, Tang H, Wang Q, et al. Gambogic acid augments black phosphorus quantum dots (BPQDs)-based synergistic chemo-photothermal therapy through downregulating heat shock protein expression. Chem Eng J. 2020;390:124312. https://doi.org/10.1016/j.cej.2020.124312.

16. Kankala R, Zhang Y, Wang S, Lee C, Chen A. Supercritical fluid technology: An emphasis on drug delivery and related biomedical applications. Adv Healthc Mater. 2017;6:1700433. https://doi.org/10.1002/adhm.201700433.

17. Palmer D, Malagari K, Kulik L. Role of locoregional therapies in the wake of systemic therapy. J Hepatol. 2020;72:277-87. https://doi.org/10.1016/j.jhep.2019.09.023.

18. Chen H, Cheng H, Wu W, Li D, Mao J, Chu C, et al. The blooming intersection of transcatheter hepatic artery chemoembolization and nanomedicine. Chinese Chem Lett. 2020;6:1375-81. https://doi.org/10.1016/j.cclet.2020.03.024.

19. Lanza E, Donadon M, Poretti D, Pedicini V, Tramarin M, Roncalli M, et al. Transarterial therapies for hepatocellular carcinoma. Liver Cancer. 2016;6:27-33. https://doi.org/10.1159/000449347.

20. Kim D, Lee J, Moon H, Seo M, Lee J, Hong S, et al. Development and evaluation of an ultrasoundtriggered microbubble combined transarterial chemoembolization (TACE) formulation on rabbit VX2 liver cancer model. Theranostics. 2021;11:79-92. https://doi.org/10.7150/thno.45348.

21. Renzulli M, Peta G, Vasuri F, Marasco G, Caretti D, Bartalen L, et al. Standardization of conventional chemoembolization for hepatocellular carcinoma. Ann Hepatol. 2021;5:100278. https://doi.org/10.1016/j.aohep.2020.10.006.

22. Savic L, Chapiro J, Funai E, Bousabarah K, Schobert I, Isufi E, et al. Prospective study of lipiodol distribution as an imaging marker for doxorubicin pharmacokinetics during conventional transarterial chemoembolization of liver malignancies. Eur Radiol. 2021;31:3002-14. https://doi.org/10.1007/s00330-020-07380-w.

23. Li A, Chan S, Thung KH. Pre-operative CT localization for patients with subsolid opacities expecting video-assisted thoracoscopic surgery-single center experience of fluorescent iodized emulsion and hook-wire localization technique. Br J Radiol. 2020;93:20190938. https://doi.org/10.1259/bjr.20190938.

24. Rho J, Lee J, Quan Y, Choi B, Shin B, Han K, et al. Fluorescent and iodized emulsion for preoperative localization of pulmonary nodules. Ann Surg. 2019;5:989-96. https://doi.org/10.1097/SLA.0000000000003300.

25. Chen H, Cheng H, Dai Q, Cheng Y, Zhang Y, et al. A superstable homogeneous lipiodol-ICG formulation for locoregional hepatocellular carcinoma treatment. J Control Release. 2020;323:63543. https://doi.org/10.1016/j.jconrel.2020.04.021. 
26. Cheng H, Yang X, Liu G. Superstable homogeneous iodinated formulation technology: Revolutionizing transcatheter arterial chemoembolization. Sci Bull. 2020;20:1685-7. https://doi.org/10.1016/j.scib.2020.06.029.

27. Gao Y, Li Z, Hong Y, Li T, Hu X, Sun L, et al. Decellularized liver as a translucent ex vivo model for vascular embolization evaluation. Biomaterials. 2020;240:119855. https://doi.org/10.1016/j.biomaterials.2020.119855.

28. Shi Z, Chu C, Zhang Y, Su Z, Lin H, Pang X, et al. Self-assembled metal-organic nanoparticles for multimodal imaging-guided photothermal therapy of hepatocellular carcinoma. J Biomed Nanotechnol. 2018;14:1934-43. https://doi.org/10.1166/jbn.2018.2636.

29. Wang H, Li X, Tse B, Yang H, Thorling C, Liu Y, et al. Indocyanine green-incorporating nanoparticles for cancer theranostics. Theranostics. 2018;8:1227-42. https://doi.org/10.7150/thno.22872.

30. Li F, Li T, Cao W, Wang L, Xu H. Near-infrared light stimuli-responsive synergistic therapy nanoplatforms based on the coordination of tellurium-containing block polymer and cisplatin for cancer treatment. Biomaterials. 2017;133:208-18.

https://doi.org/10.1016/j.biomaterials.2017.04.032.

31. Tanaka T, Masada T, Nishiofuku H, Fukuoka Y, Sato T, Tatsumoto S, et al. Development of pumping emulsification device with glass membrane to form ideal lipiodol emulsion in transarterial chemoembolization. Eur Radiol. 2018;28:2203-7. https://doi.org/10.1007/s00330-017-5197-x.

32. Du J, Li H, Wang J. Tumor-acidity-cleavable maleic acid amide (TACMAA): A powerful tool for designing smart nanoparticles to overcome delivery barriers in cancer nanomedicine. Acc Chem Res. 2018;51:2848-56. https://doi.org/10.1021/acs.accounts.8b00195.

33. Miyashiro I, Kishi K, Yano M, Tanaka K, Motoori M, Ohue M, et al. Laparoscopic detection of sentinel node in gastric cancer surgery by indocyanine green fluorescence imaging. Surg Endosc. 2011;25:1672-6. https://doi.org/10.1007/s00464-010-1405-3.

34. Lei Y, Zeng L, Xie S, Fan K, Yu Y, Chen J, et al. Sertraline/ICG-loaded liposome for dual-modality imaging and effective chemo-photothermal combination therapy against metastatic clear cell renal cell carcinoma. Chem Biol Drug Des. 2020;95:320-31. https://doi.org/10.1111/cbdd.13652.

35. Fontoura OA, Ferreira H. Neovagina creation in congenital vaginal agenesis: New mini-laparoscopic approach applying intraoperative indocyanine green fluorescence. Surg Innov. 2020;2:1-9. https://doi.org/10.1177/1553350620968990.

36. Lieto E, Galizia G, Cardella F, Mabilia A, Basile N, Castellano P, et al. Indocyanine green fluorescence imaging-guided surgery in primary and metastatic liver tumors. Surg Innov. 2018;25:62-8. https://doi.org/10.1177/1553350617751451.

\section{Figures}




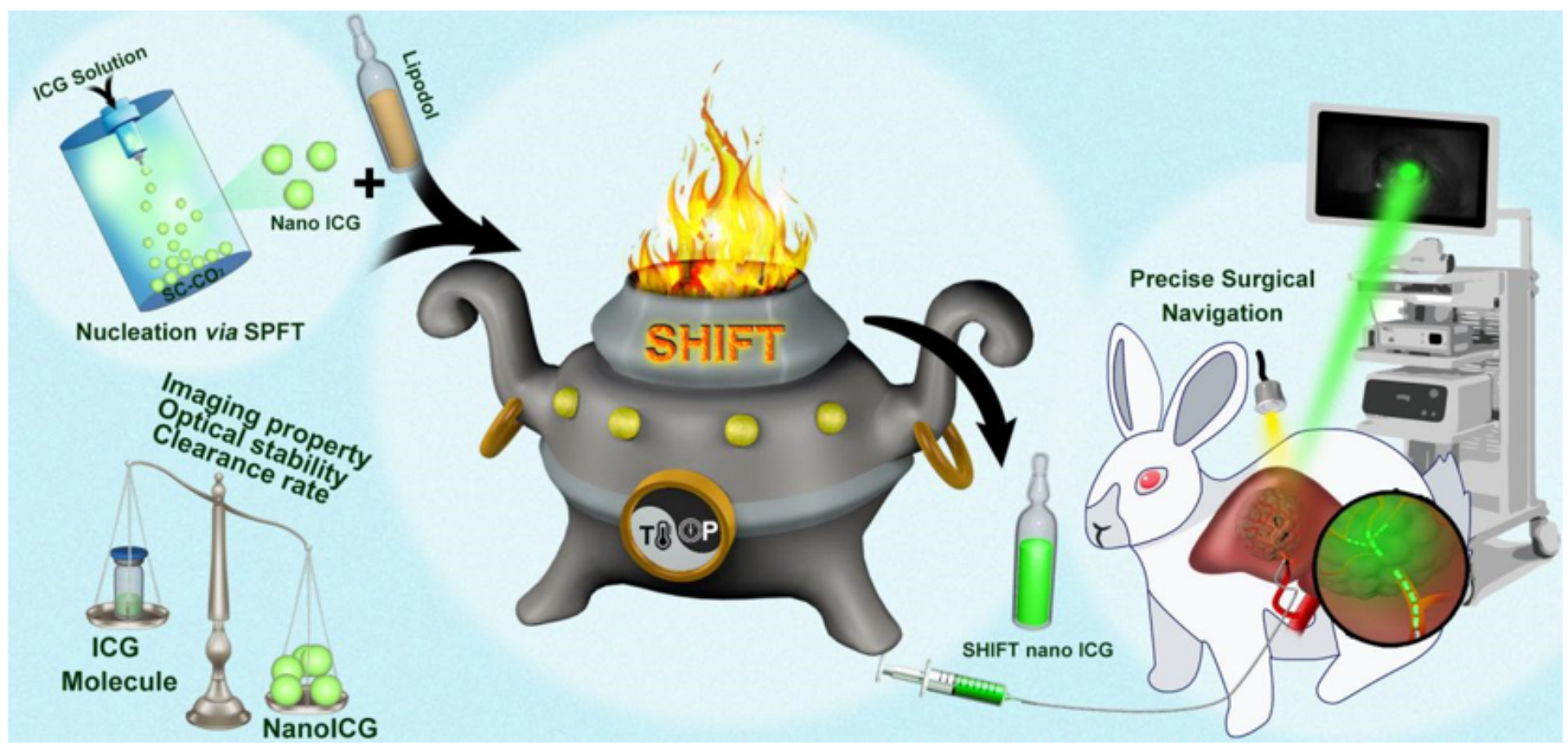

\section{Figure 1}

Schematic illustration of SHIFT nanoICG preparation and fluorescence-guided precise hepatectomy. Super-stable pure-nanomedicine formulation technology (denoted as SPFT) was employed to produce nanoICG with enhanced imaging property, anti-photobleaching capacity, and retention ability in tumor regions compared with ICG molecule. Subsequently, nanoICG was homogenously dispersed into lipiodol via SHIFT for specific deposition into hepatocellular carcinoma lesions through transcatheter embolization, and then achieving precise fluorescence-guided resection. 
a

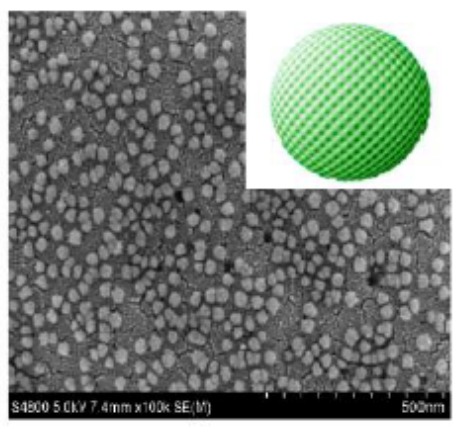

b



C



d



Free ICG $(\mu \mathrm{g} / \mathrm{mL})$ nanolCG $(\mu \mathrm{g} / \mathrm{mL}) \quad \mathrm{g}$

f e

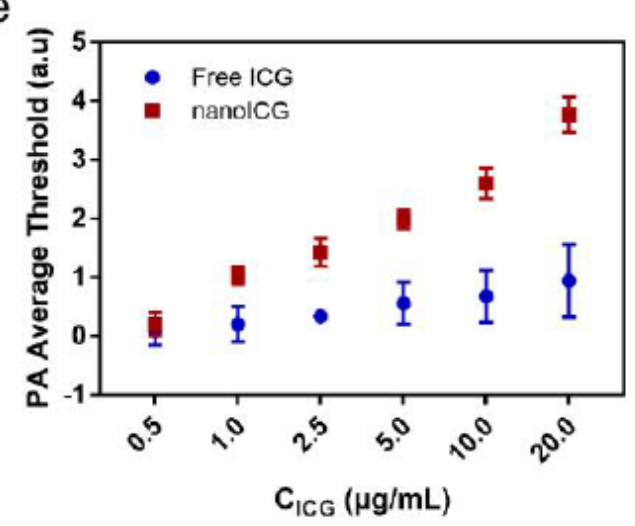

Laser $0 \mathrm{~min}$

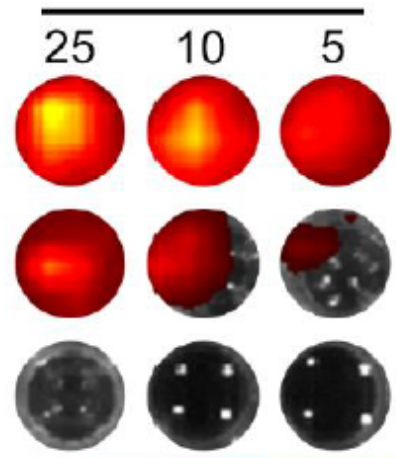

Min

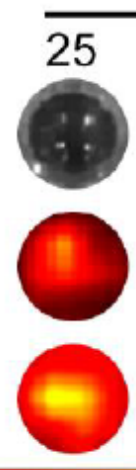

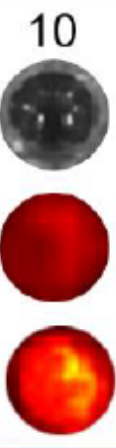

2)

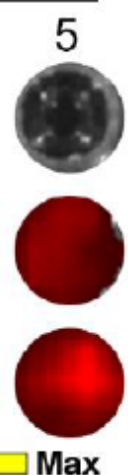

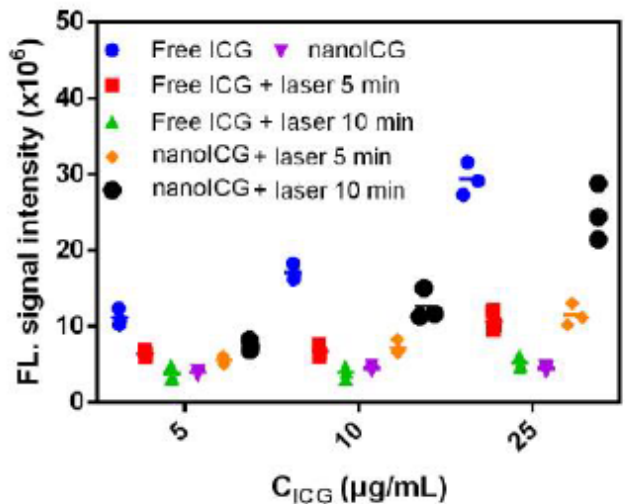

Figure 2

Characterization of nanoICG. a Representative SEM image of nanolCG. Scale bar: $500 \mathrm{~nm}$. b DLS of nanoICG. c Free ICG and nanoICG ultraviolet absorption spectra. d, e The photoacoustic signal of free ICG and nanoICG at different concentrations (d), and the quantitative analysis of free ICG and nanoICG (e). f, $\mathrm{g}$ Anti-photobleaching experiments of free ICG and nanoICG ( $\mathrm{f})$, and the quantitative analysis $(\mathrm{g})$. 
a

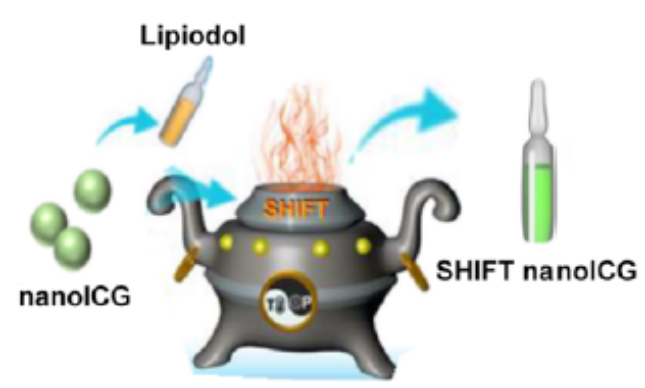

d

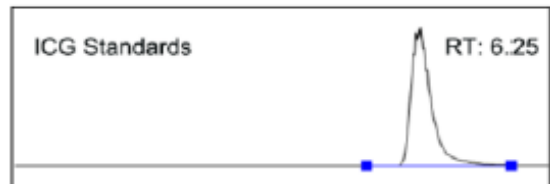

SHIFT nanoICG

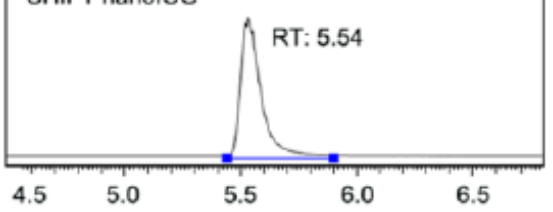

g

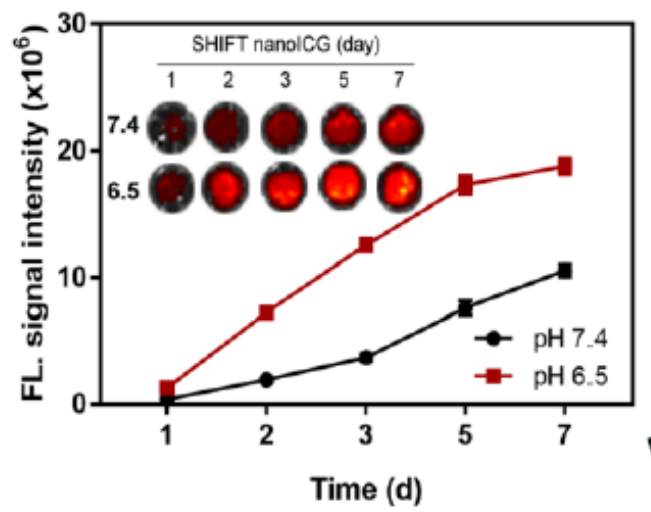

b

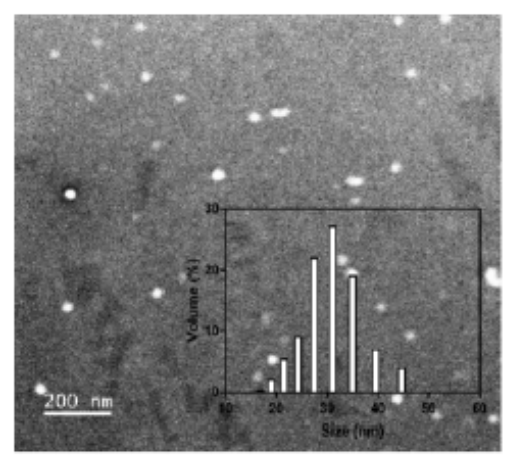

e

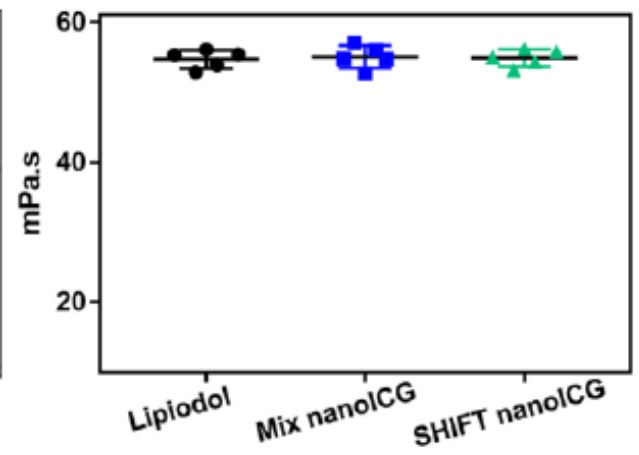

h
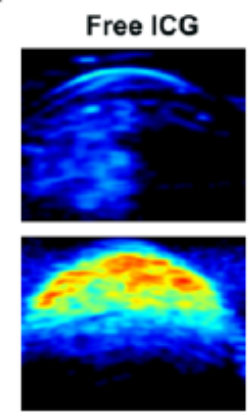

Mix nanolcG
SHIFT nanoClG

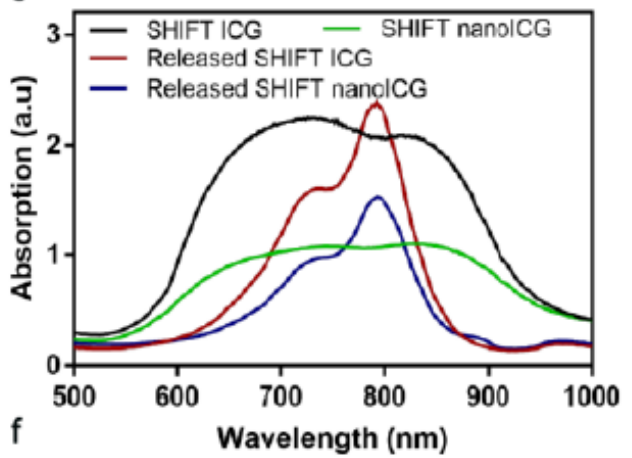

f Wavelength $(\mathrm{nm})$



i

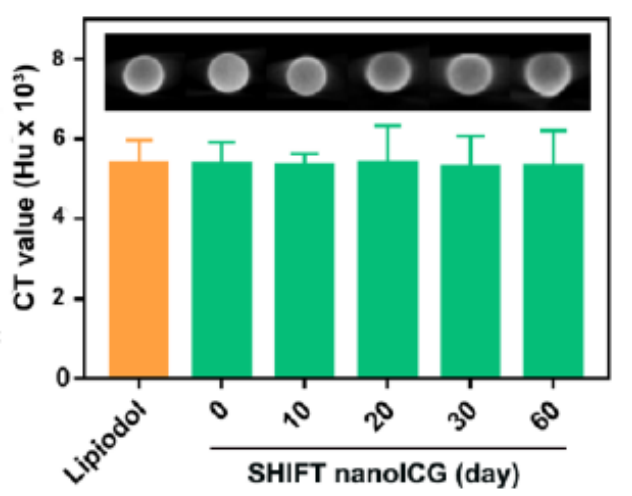

\section{Figure 3}

Preparation and characterization of SHIFT nanoICG. a Schematic of SHIFT nanolCG preparation. b Representative TEM image of released nanoICG (Inset: DLS of released nanolCG). c The ultraviolet absorption spectra of SHIFT ICG, SHIFT nanoICG, released ICG, and released nanolCG. d LC-MS of SHIFT nanolCG. e The viscosity of lipiodol, mixture of nanoICG and lipiodol, and SHIFT nanolCG. $f$ The photograph of the mixture of nanoICG and lipiodol, and SHIFT nanolCG of newly prepared and stored after 60 days. g Fluorescent images and signals of SHIFT nanolCG in the condition of $\mathrm{pH} 6.5$ and $\mathrm{pH} 7.4$. h Photoacoustic images and signals of free ICG, nanoICG, mix nanolCG, and SHIFT nanolCG. i CT images and signals of lipiodol and SHIFT nanoICG. 
a

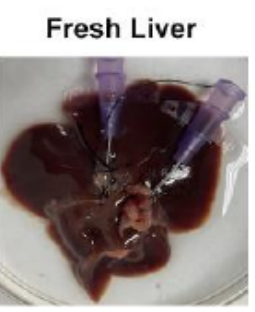

b
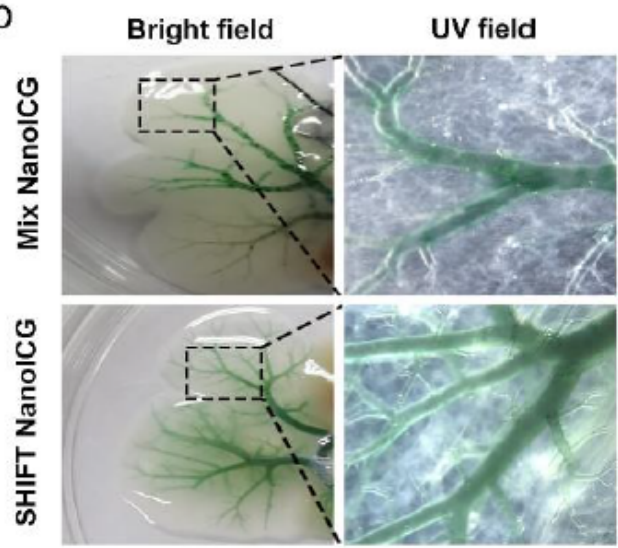

c


e
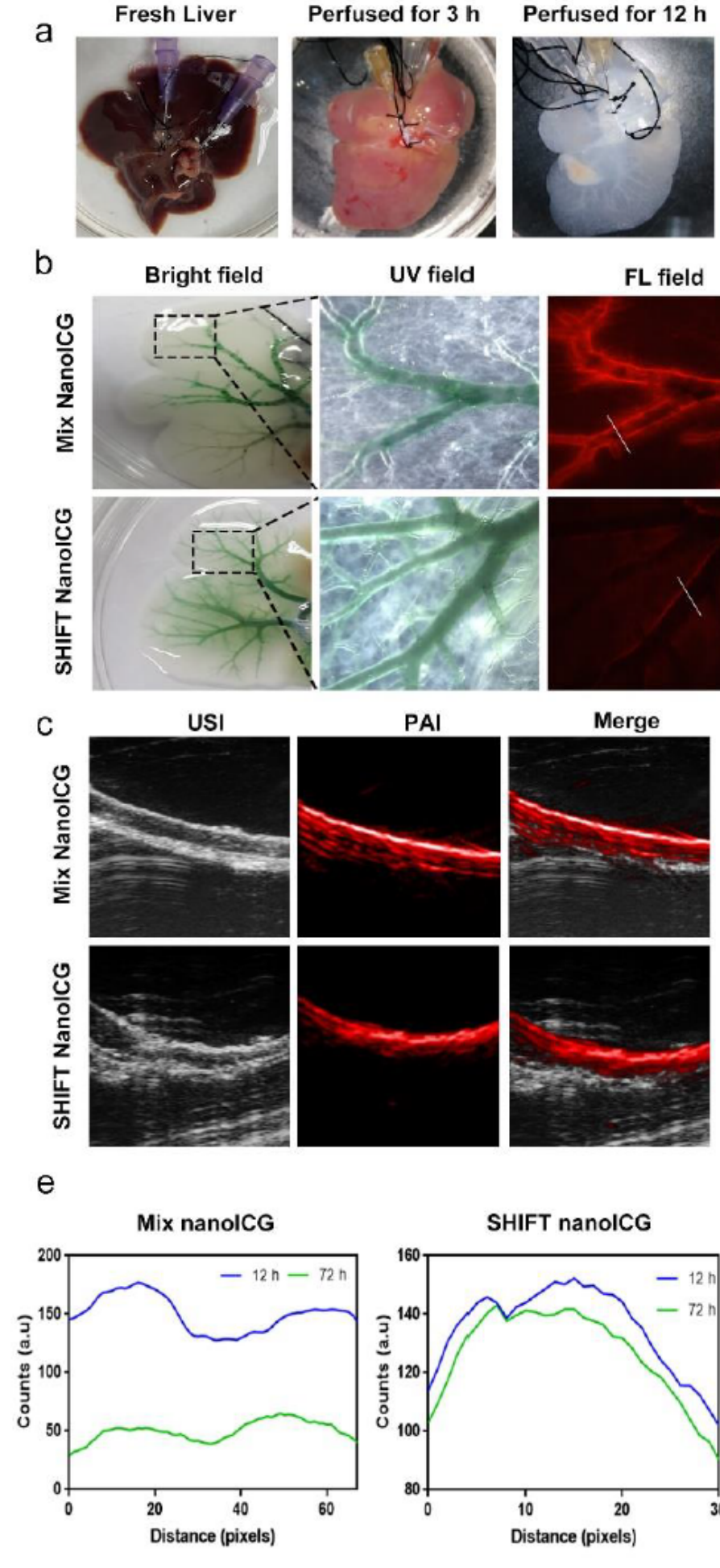

FL field

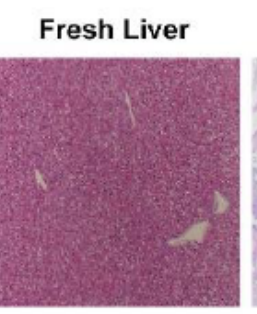

Perfused for $\mathbf{3 ~ h}$

Perfused for $12 \mathrm{~h}$
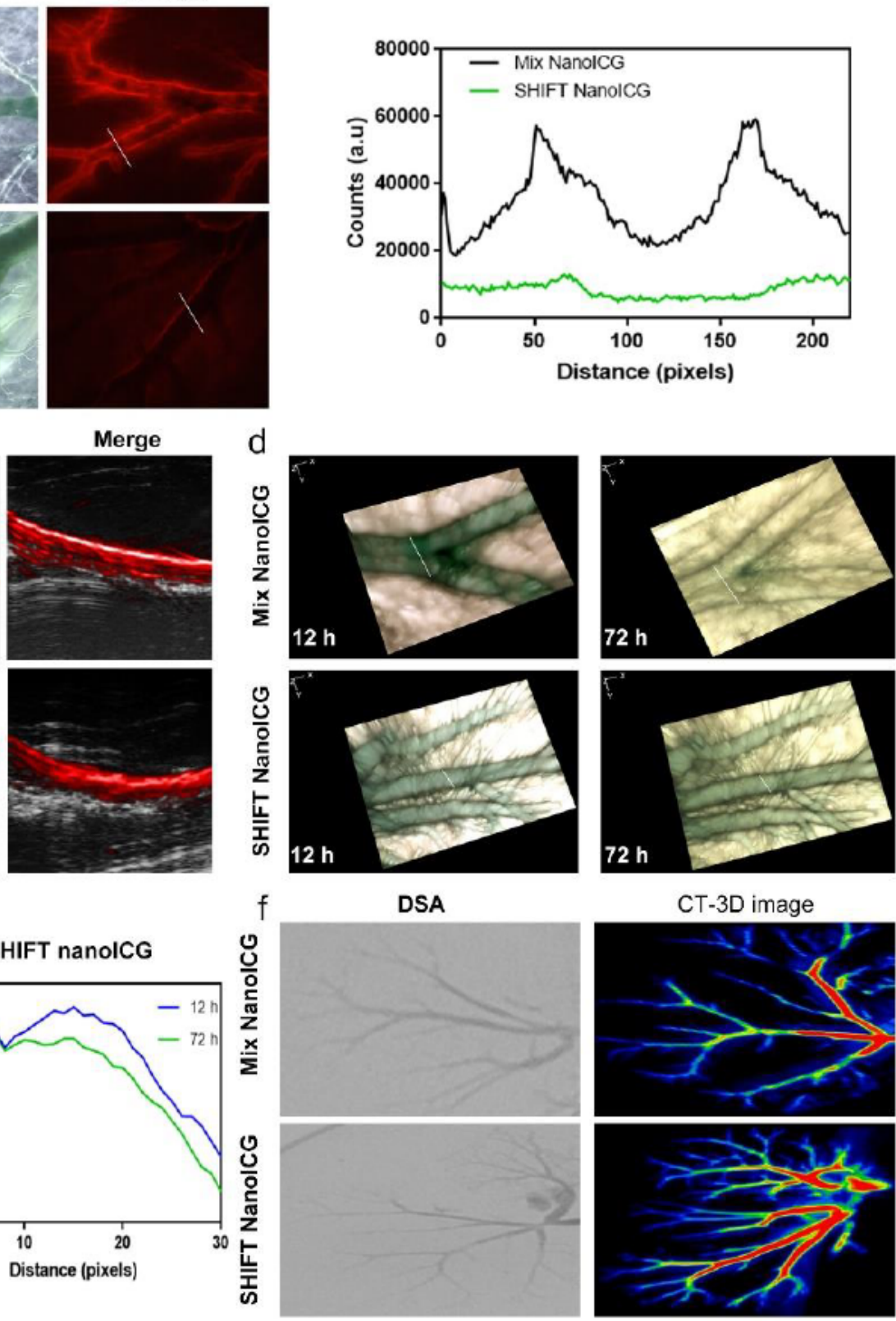

\section{Figure 4}

Preparation and characterization of SHIFT nanoICG in decellularized liver model. a Identification of decellularized liver model. b The stereoscope images of decellularized liver models and semi-quantitative analysis of fluorescence intensity at each time point base on the location of white line after $24 \mathrm{~h}$ injection of SHIFT nanoICG and Mix nanoICG. c The photoacoustic images of decellularized liver models after 24 h injection of SHIFT nanoICG and Mix nanolCG. d Representative 3D confocal microscopy images of 
nanoICG released from Mix nanoICG and SHIFT nanoICG formulation after $12 \mathrm{~h}$ and $72 \mathrm{~h}$ injection. $\mathrm{e}$ Semi-quantitative analysis of fluorescence intensity at each time point base on the location of white line $12 \mathrm{~h}$ and $72 \mathrm{~h}$ fluorescent vision. $\mathrm{f}$ Representative CT-3D images of lipiodol in venous system of decellularized liver model after $72 \mathrm{~h}$ injection.

a

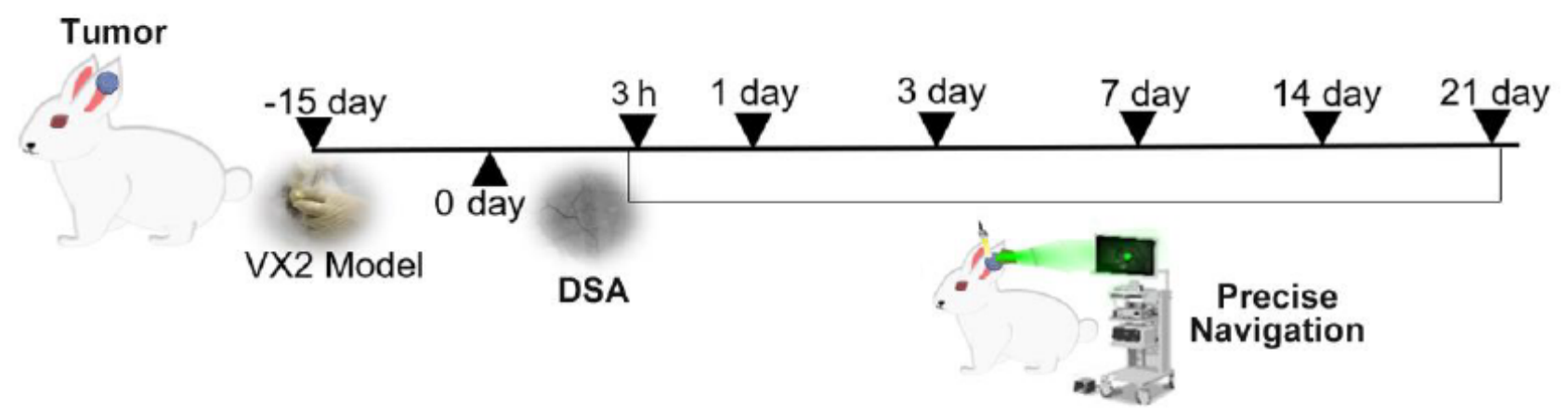

b
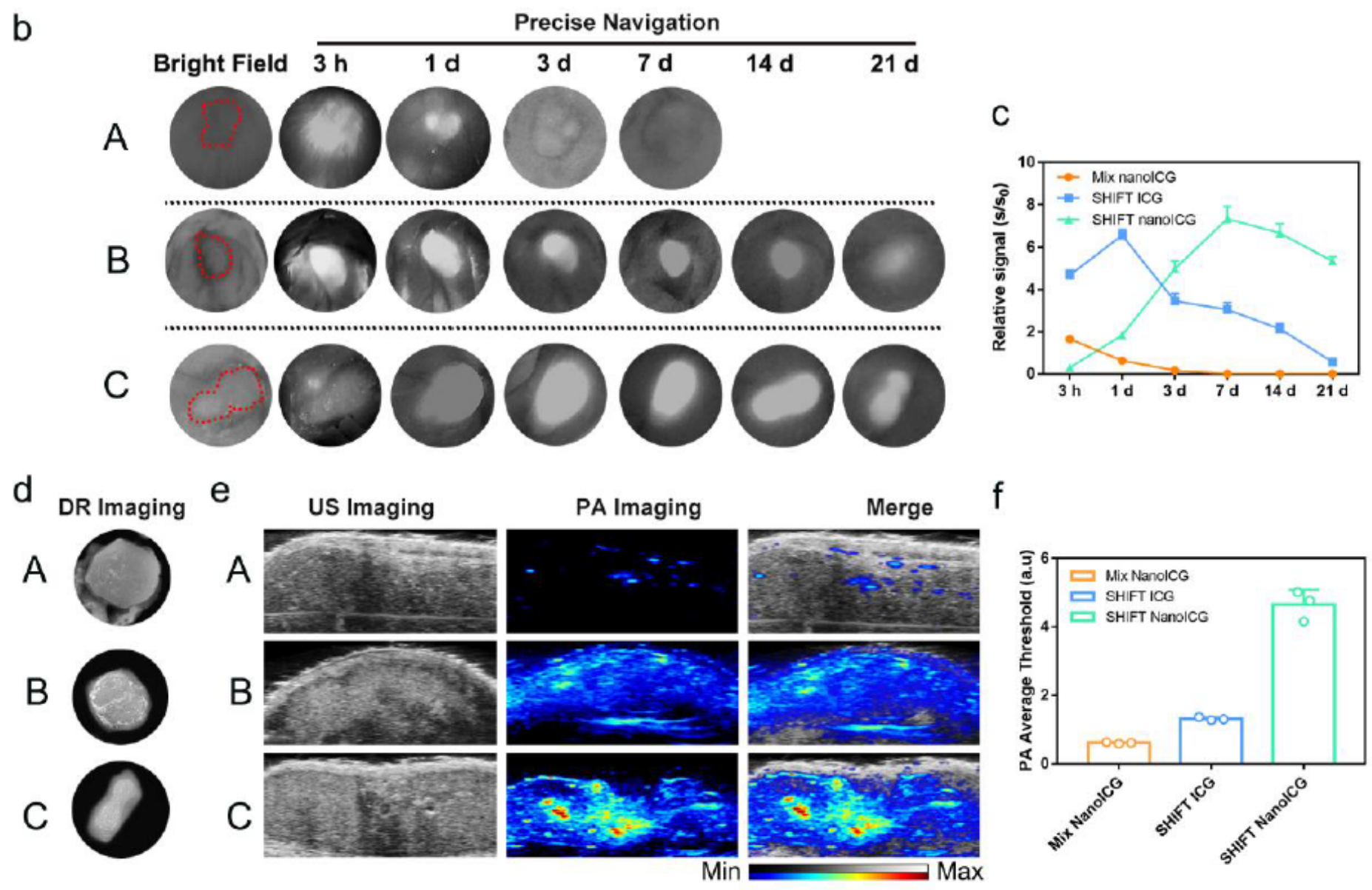

\section{Figure 5}

The navigation performance of SHIFT nanoICG in rabbit ear-bearing VX2 subcutaneous models. a Schematic illustration of fluorescent surgical navigation trail by SHIFT nanoICG. b Continuous navigation performance monitoring of mix nanoICG (A), SHIFT ICG (B), and SHIFT nanoICG (C) for 21 days. C Quantitative analysis of navigation performance. $d$ DR imaging of the resected VX2 lesions. e, $f$ PA 
imaging of the resected lesions (e) and the quantitative analysis (f). A, B and C represent mix nanolCG, SHIFT ICG, and SHIFT nanoICG groups respectively.
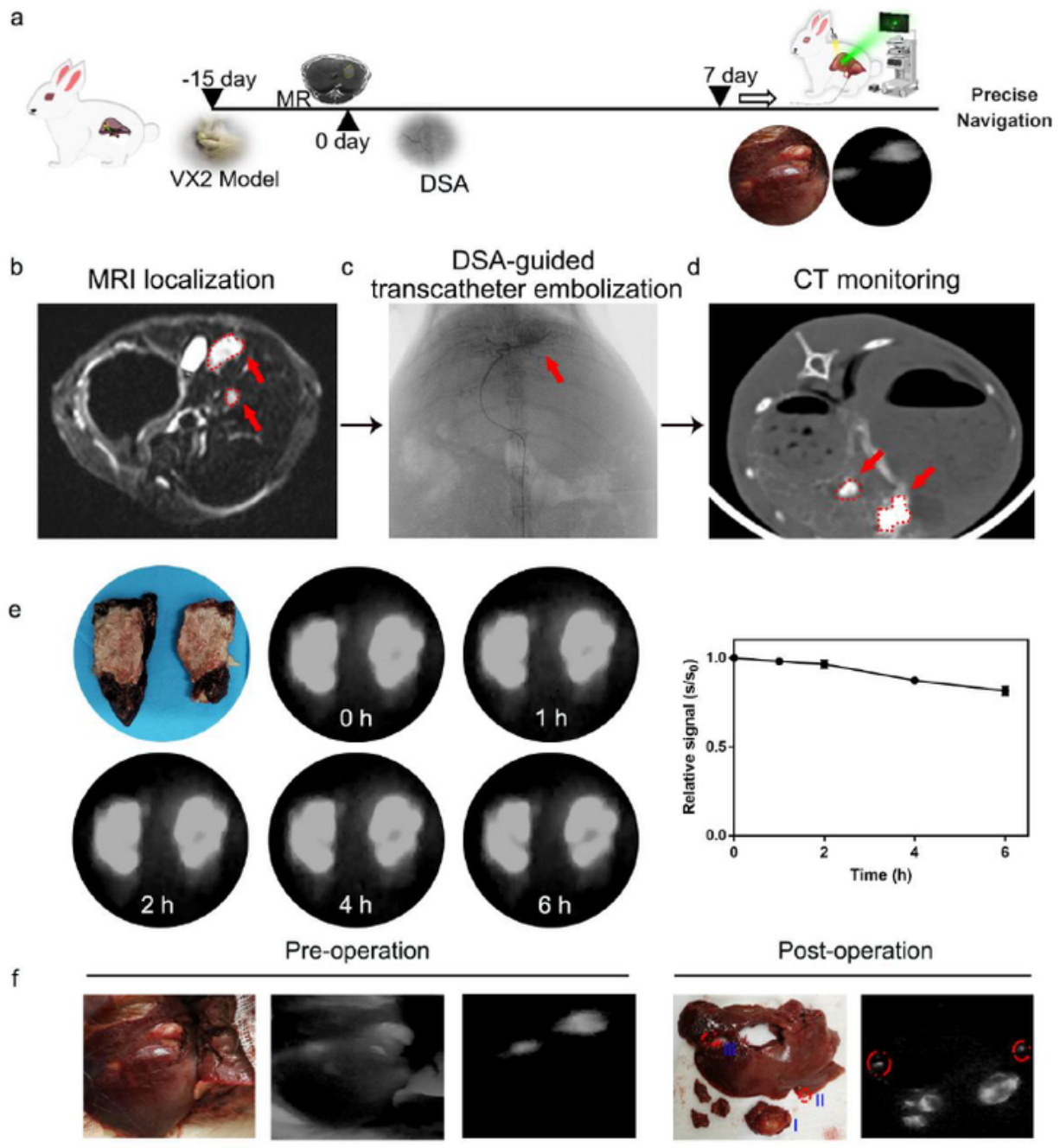

g

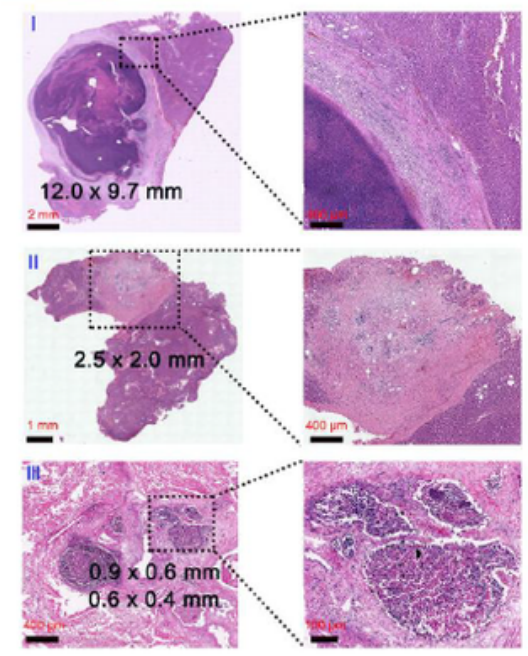

h

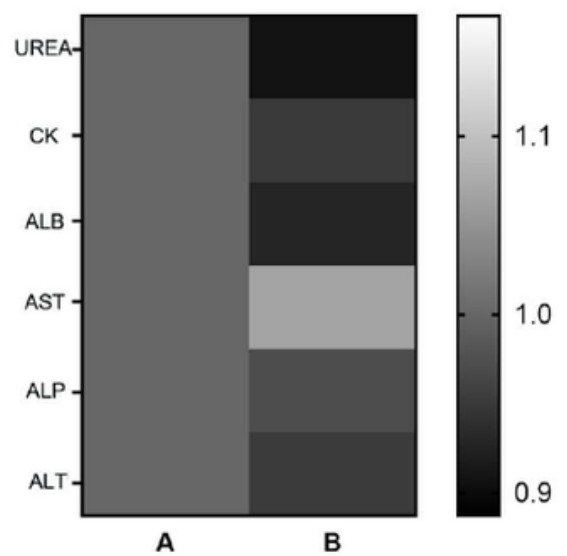

Figure 6

The evaluation of fluorescence-guided surgical navigation of SHIFT nanoICG in orthotopic HCC models. a Schematic illustration of fluorescent surgical navigation trail by SHIFT nanoICG. b-d Orthotopic models verified via MRI (b), DSA guided embolization of SHIFT nanoICG (c), and CT monitored embolic 
evaluation (d). e The anti-photobleaching was recorded underexposure of navigation system. $f$ Fluorescence-guided orthotopic VX2 model resection. $g$ H\&E staining of representative resected tumor lesions. $h$ The liver and kidney function measurement of control (A) and SHIFT nanolCG group (B).

\section{Supplementary Files}

This is a list of supplementary files associated with this preprint. Click to download.

- SupplymentaryInformationnanolCGlipiodolformulation825.docx 